\title{
How to Implement Lightweight Threads
}

\author{
Mauricio Toro \\ Universidad EAFIT
}

January 5, 2019

\section{Introduction}

In computer science, a thread represents a sequential flow control or an abstraction of a processor, a continuation is an abstraction of the processor registers and the events are an abstraction of the hardware interruptions.

Sometimes, threads are described by their weight, meaning how much contextual information must be saved for a given thread in order to schedule them [WGQH].

For example, the context of a Unix process includes the hardware register, the kernel stack, user-level stack, process id, and so on. The time required to switch from one Unix process to another is large (thousands of microseconds), for that reason those are called heavyweight threads.

Modern operating systems kernels, such as Mac OS X and Mach, allow to have multiple threads in the same process, decreasing the amount of context that must be saved with each one. These threads are called medium weight threads and it takes hundreds of microseconds to switch between them $\left[\mathrm{TRG}^{+} 87\right]$.

When all context and thread operations are exposed at user level, each application needs only a minimal amount of context information saved with it, so that context switching can be reduced to tens of microseconds. These are called lighweight threads.

For example, Lightweight threads used by the Java VM outperform linux threads on thread activation and synchronization because thread management operations do not need to cross kernel protection boundaries. But, linux native threads have better performance on I/O operations [App96].

Since Lightweight threads may block all the other threads when perform a blocking I/O operation, it is necessary to use asynchronous I/O operations, adding complexity that increases latency for I/O operations. 
Nevertheless, Lightweight threads enable an application to use a thread management system that is more appropriate to the problem domain [MEG94]. Table 1 gives an example of the performance of them.

\begin{tabular}{|c|c|c|c|}
\hline Type of threads & Lightweight & Medium weight & Heavyweight \\
\hline Creation Time & 52 & 350 & 1700 \\
\hline $\begin{array}{c}\text { Synchronization } \\
\text { with semaphores }\end{array}$ & 66 & 390 & 200 \\
\hline
\end{tabular}

Table 1: Technical report on SPARCstation 2 - Time in microseconds [Vah96]

Strategies to implement Lightweight threads include, but are not limited to Event driven programming, Scheduler activations, Protothreads, Virtual machine with thread support, Coroutines, Continuations and Multiple stack based threads.

\section{Event driven programming}

Event-based programs are typically driven by a loop that polls for events and executes the appropriate callback when the event occurs. This means that the flow of the program is determined by sensor outputs, user actions or messages from other programs.

They tend to have more stable performance under heavy load than threaded programs according to $\left[\mathrm{DZK}^{+} 02\right]$. However, there are problems when they are used with synchronous I/O operations. Being necessary to use asynchronous I/O .

In the other hand, Ron von Behren et al [vBCB03] argue that although Event-based programs have been used to obtain good performance in high concurrency systems, if there is a good implementation of threads, with a tight integration with the compiler, it is possible to obtain similar o higher performance with threads than with events.

In order to implement this model it is required: a dispatcher, which takes the events and call the appropriate handler; an event queue, which stores the events when the dispatcher is busy; and different handlers for each type of events [Fer06] . The diagram of figure 1 represents this model.

When the events can change the "state" of the program, it is necessary to have a Finite State Machine (FSM) to keep track of the current state of the program [Fer06]. 


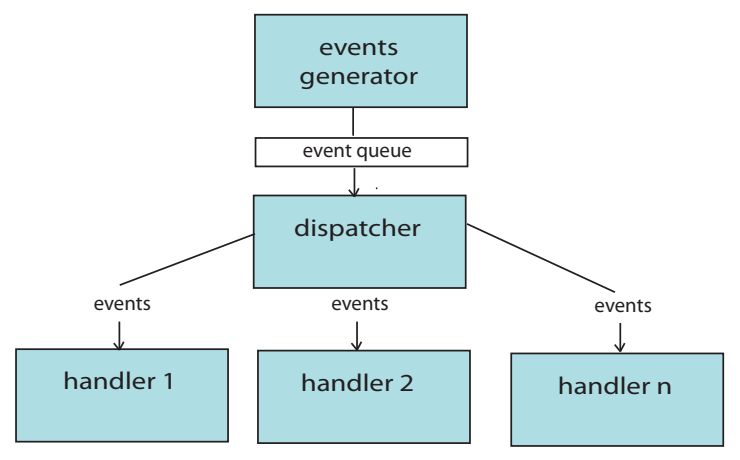

Figure 1: Event Driven Programming Control Flow

\section{Scheduler activations}

Scheduler activations [ABLL90] is a threading mechanism that maps N user level threads into some M kernel threads. This takes the advantages from the kernel-level ("1:1") and the user-level ("N:1") threading. Scheduler activations for Linux $O S$ were implemented in two modules: a patch for the linux kernel and the user-level part was developed by the Informatics Research Laboratory of Bodeaux (LABRI) in a library called Marcel threads $\left[\mathrm{BDT}^{+} 08\right]$.

\section{Coroutines}

A coroutine is a non-preemptive thread. They generalize subroutines to allow multiple entry points, suspending and resuming execution at certain locations [Knu73].

In the $\mathrm{C}$ language there is a library called the Portable Coroutine Library (libpcl), high order programming languages such as Python and Ruby have support for them also. Scheme coroutines are made using continuations, which are a functional object containing the state of the computation. When the object is evaluated, the store computation is restarted where it left off [Gra04]. 


\section{Protothreads}

Protothreads [DS05] propose an abstraction that reduces the complexity of Event-based programs. Using protothreads, it is possible to perform conditional blocking on top of Event-based programs, without the overhead of multi-threading which includes multiple stacks. They use local continuations, which behaves like continuations, but they do not save the stack.

\section{Virtual machines with thread support}

There are virtual machines with thread support such as the Simple Extensible Abstract Machine (SEAM) [BK02]. SEAM has been used to implement a naive Java Virtual Machine and the Alice language [Kor01]. SEAM supports the concurrent execution of multiple threads in the traditional way: there is a stack of activation records, each record correspond to task to be executed. The scheduler coordinates the execution of multiple threads and the preemption. There are too disadvantages with $S E A M$ : When a thread is blocked in a function outside $S E A M$, all the other threads get blocked too and it does not provide good abstractions for implementing a system that needs to be concurrent with respect to external processes. [Tac08]

\section{Multiple stack based threads}

Each thread is represented by using an structure that contains thread ID, execution context, priority and the thread stack. Additionally, there is an scheduler to allow the concurrent execution of all threads [SS92].

\subsection{Thread Schedulling}

The scheduler is in charge of providing a fair execution to all threads, a fair execution means that every thread will eventually execute. There is a runnable pool containing the runnable threads, when a new thread is created it is added to the runnable pool. There is also a suspended pool, where the threads that are "waiting" remain until their waiting condition becomes true. Finally, there are a terminated pool and the current thread([Sch02], [VH04]). Figure 2 explains the thread states and their transitions .

There are many scheduling policies such as Priorities, First Come First Served (FCFS), Shortest Process Next (SPN), Shortest Remaining Time (SRT) and Round Robin [PS06]. We will focus in Round Robin, which makes 


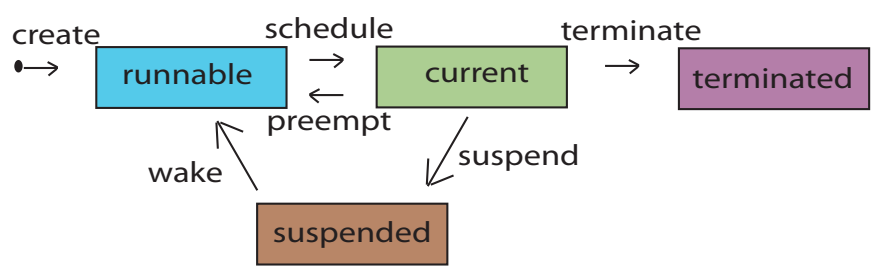

Figure 2: Thread states and their transitions [Sch02]

a fair execution to all threads, it is commonly used to model interactive systems. It keeps all the threads in a queue and it guarantees that processor time is put equitably over the threads.

The time slice given to each thread cannot be too small because it will cause an overhead of queue management, however it cannot be too large because it will not be useful for interactive systems. There is another approach, the counting approach; which counts computation steps and give the same number of them to each thread, it is often used in real time systems [VH04].

Priorities are important when we need to give more processor time to some threads than others. But, high priority threads should not starve low priority threads.

When we want to mix priorities and Round Robin, there are different possible approaches: processes on an equal priority are addressed in a roundrobin manner; the time slice duration can change according to the priority; and the one used by Mozart $O z$, where every tenth time slice of a high priority thread, a medium priority thread is give one slice and similarly with the medium and low priority threads [VH04].

When a thread creates a child thread, the child is given the same priority as the parent to avoid timing bugs such as the priority inversion, which is an scenario where a low priority task holds a shared resource that is required by a high priority task, causing the delayed execution of the high priority task, this problem happened in the Mars' exploration mission [Ree05].

\subsection{Synchronization}

Multiple solutions have been proposed for synchronization, such as: semaphores, monitors, message-passing, locks, etc. All them accesses to a shared state, 
making very difficult to write correct concurrent programs. Another approach is declarative concurrency [Uni03].

It consists of several threads of statements executing concurrently and communicating via a shared store, the store only contains logic variables. Those variables have two states: bound and not bound. When a variable is required for a computation and it has not been bound, the thread yields until the variable is bound, this way the synchronization is achieved.

\section{How to implement lightweight threads in Com- mon Lisp}

\subsection{Continuations}

It is possible to simulate concurrent threads using continuations and UNIX signals to provide preemption. The continuation of each thread must be saved. This way they can be invoked at a later time. When a thread must block, we can capture the continuation using the $c a l l / c c$ macro, provided by libraries such as cl-cont, since continuation are not natively implemented in common lisp. This approach has been used to implement a concurrent version of $M L$ called $S M L / N J$ [CM90].

A problem of using continuations is that they capture only the state that describes the processor, but they do not capture the state of the I/O systems [Shi97].

Another issue about using continuations is the lack of natively implementation in Common Lisp. Even though the continuation passing style $(C P S)$ can be obtained writing lisp macros, it creates an overhead, leading to high memory and time consumption. Tables 2 and 3 describes the memory and time consumption of a function which adds all the elements of a list. Data was obtained after several tests under Mac OS 10.5 using an Imac Intel Core 2 duo $2.8 \mathrm{ghz}$, LispWorks Professional 5.02 and SBCL 1.012. Lispworks performance is not very good for $C P S$ style code, probably because the compiler is not optimized to handle CPS style code.

\subsection{Event-driven programming}

Each program will be written as an event loop, which runs by taking an event and executing some code, depending of the type of event, and then posting one or more new events. Lightweight threads can be done this way, having several event queues. The scheduler picks one event from one queue to execute each time around. 


\begin{tabular}{|c|c|c|}
\hline Normal code & Lispworks & SBCL \\
\hline Time consumption & 0.002 & 0.001 \\
\hline Memory consumption & N/A & 81920 \\
\hline CPS code & & \\
\hline Time consumption & 0.056 & 0.008 \\
\hline Memory consumption & 3453000 & 1437824 \\
\hline
\end{tabular}

Table 2: List of size 5000 (time in seconds, memory in bytes)

\begin{tabular}{|c|c|c|}
\hline Normal code & Lispworks & SBCL \\
\hline Time consumption & 0.004 & 0.002 \\
\hline Memory consumption & 5492 & 320466 \\
\hline CPS code & & \\
\hline Time consumption & 0.242 & 0.018 \\
\hline Memory consumption & 13600736 & 5763064 \\
\hline
\end{tabular}

Table 3: List of size 20000 (time in seconds, memory in bytes)

An event is simply a data structure. For example, it can be a record representing a mouse click, with the coordinates or the window in which the click happened. It can also be something inside the application. If the application does a very long calculation, then it can be a good idea to split the calculation into smaller ones. Each small one then sends an event to start the next small one. This keeps the application from hogging the processor.

In order to achieve thread synchronization, it is possible to add a wait and a bind event for waiting until a variable takes is bound and for binding variables. Asynchronous send and blocking receive can be achieved assigning a mailbox to each thread ${ }^{1}$ [Roy08].

\subsection{Making an scheduler in $\mathrm{C}$}

Gambit- $C^{2}$ and $C M U-C L$ have successfully implemented Lightweight threads directly in the language sources. This cannot be done when the Common Lisp distribution is non-free.

The use of native threads to implement first-class continuations in the

\footnotetext{
${ }^{1}$ Lispworks have an API for mailboxes already implemented

${ }^{2}$ Gambit-C is an Scheme distribution which implements lighweight threads efficiently
} 
Scheme thread system, to allow arbitrary nesting of calls between Scheme and $C$, has many restrictions and overheads in most $C$ thread systems according to [Fee00]. These results probably could be generalized to Common Lisp.

\section{Example of Event Driving Programming in Com- mon Lisp}

The example presented in this section if an interpreter for Concurrent Constraint Programming (CCP) [SMP91]. The program control is a main loop, handling different events, which are taken from several event queues. Those event queues represent the Lightweight threads.

We have 3 types of events: ask, tell and parallel composition. The tell handler calls a Gecode [Sch02] propagator, the parallel handler creates one

thread (event queue) for each process. Finally, the ask handler, test if the variables required for the condition are assigned. If they are assigned, it creates a new event with the "ask's body", else it adds to the queue the same ask event again. Notice that in Gecode there is a non-blocking operation to ask if a variable has been assigned. Figure 3 describes the architecture used for the Concurrent Constraint Programming (CCP) interpreter.

\subsection{Finding if there is a path between 2 vertices in a Graph}

The idea behind this processes is having one $C C P$ process for each arc. Each Arc_Process $(i, j)$ sends Forward signals to its successors and Back signals to its predecessors. When an Arc_Process $(i, j)$ receives a back signal and a forward signal it tell the store that there is a path.

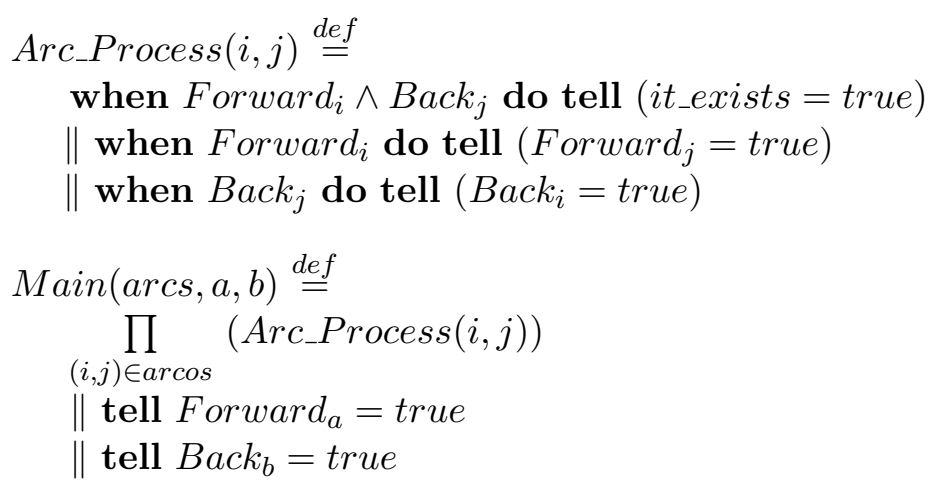

These two $C C P$ agents can be easily represented, using lisp structures, in 
the event driven $C C P$ interpreter. The tell processes are represented with structures containing the propagator function and its arguments; the parallel structures contain a list of events; and the ask processes contain the variables used in the ask relation, the relation between those variables and the event representing the "ask's body". Notice that syntax could be easily simplified using Lisp Macros.

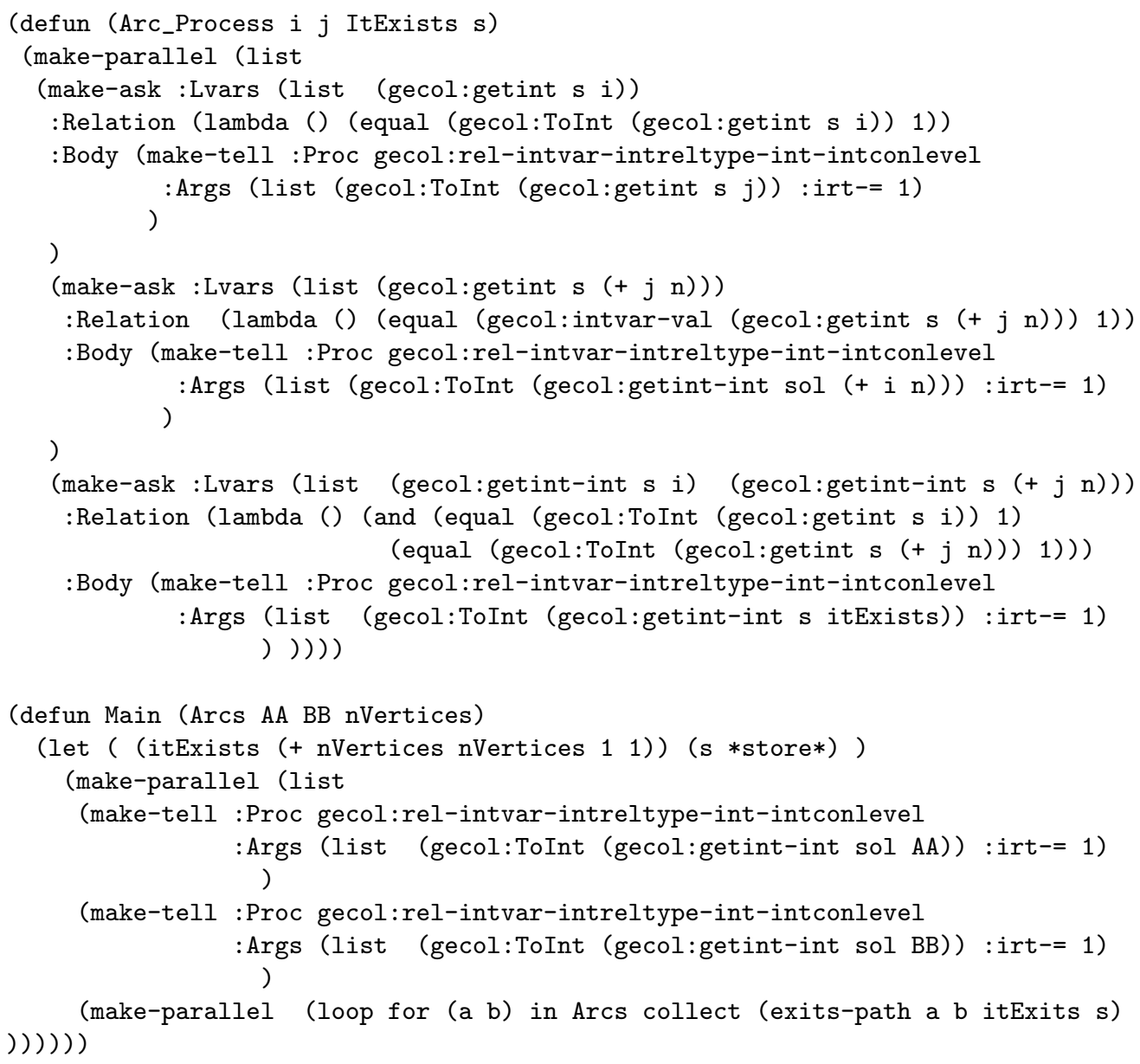




\section{Conclusions}

Using continuations in high order languages such as Common Lisp is not very efficient because they do not work close to the compiler. Other options such as making an scheduler in $C$ can be efficient when the sources of the interpreter are available and they can be modified, otherwise, making an interface with $C$ using FFI causes an overhead, making it impossible to achive "lighweight".

To make an efficient implementation of lighweight threads in Common Lisp it is required to analyze the type of applications which will use the threads. For example, for a Concurrent Constraint Programming (CCP) interpreter using Gecode, event driven programming seams very natural, but for the Omax system $\left[\mathrm{ABC}^{+} 06\right]$, it could not be appropriate.

Process calculi have been applied to the modeling of interactive music systems [Tor18b, Tor18a, Tor18h, Tor18d, TDCJO18, Tor18f, Tor18g, Tor18c, Tor18e, Tor16b, TDCC16, TRAA16, Tor16a, TRAA15, ADCT11, TDCR14, TAAR09, $\mathrm{ORS}^{+}$11, Tor12, Tor09a, Tor10, Tor15, $\mathrm{AAO}^{+}$09, TDCC12, Tor09b, TDC10, TDCB10, Tor08] and ecological systems [TPA ${ }^{+} 16, \mathrm{PT} 13$, TPKS14, PTA13]. In addition, research on algorithms [PFATT16, MPT17, RPT17, QSRT18, RHRS ${ }^{+}$18] and software engineering [STM $\left.{ }^{+} 18, \mathrm{MTC}^{2}\right]$ also contributes to this field.

\section{Future Work}

- Compare efficiency of "traditional" Lispworks processes Vs simple lispworks processes

- Compare efficiency of the concurrent program for finding a path in graph running in Lispworks Vs a prototype developed in Mozart Oz.

- Evaluate the alternative proposed for concurrency control in the Abstract Machine for Pico calculus (Mapico) [ABT99].

\section{References}

$\left[\mathrm{AAO}^{+} 09\right]$ Jesús Aranda, Gérard Assayag, Carlos Olarte, Jorge A. Pérez, Camilo Rueda, Mauricio Toro, and Frank D. Valencia. An overview of FORCES: an INRIA project on declarative formalisms for emergent systems. In Patricia M. Hill and 
David Scott Warren, editors, Logic Programming, 25th International Conference, ICLP 2009, Pasadena, CA, USA, July 1417, 2009. Proceedings, volume 5649 of Lecture Notes in Computer Science, pages 509-513. Springer, 2009.

$\left[\mathrm{ABC}^{+} 06\right]$ Gérard Assayag, Georges Bloch, Marc Chemillier, Arshia Cont, and Shlomo Dubnov. Omax brothers: a dynamic yopology of agents for improvization learning. In $A M C M M$ '06: Proceedings of the 1st ACM workshop on Audio and music computing multimedia, pages 125-132, New York, NY, USA, 2006. ACM.

[ABLL90] Thomas E. Anderson, Brian N. Bershad, Edward D. Lazowska, and Henry M. Levy. Scheduler activations: Effective kernel support for the user-level management of parallelism. Technical Report 90-04-02, Seattle, WA (USA), 1990.

[ABT99] Mauricio Heredia Antal Buss and Gabrierl Tamura. Mapico: maquina abstracta para el calulo pico, 1999.

[ADCT11] Antoine Allombert, Myriam Desainte-Catherine, and Mauricio Toro. Modeling temporal constrains for a system of interactive score. In Gérard Assayag and Charlotte Truchet, editors, Constraint Programming in Music, chapter 1, pages 1-23. Wiley, 2011.

[App96] For Embedded Applications. Comparative performance evaluation of java threads, 1996.

$\left[\mathrm{BDT}^{+} 08\right]$ Franois Broquedis, Franois Diakhat, Samuel Thibault, Olivier Aumage, Raymond Namyst, and Pierre-Andr Wacrenier. Scheduling dynamic openmp applications over multicore architectures. In International Workshop on OpenMP (IWOMP), West Lafayette, IN, May 2008. To appear.

[BK02] Thorsten Brunklaus and Leif Kornstaedt. A virtual machine for multi-language execution. Submitted, November 2002.

[CM90] Eric C. Cooper and J. Gregory Morrisett. Adding threads to standard ML. Technical Report CMU-CS-90-186, Pittsburgh, PA, 1990.

[DS05] A. Dunkels and O. Schmidt. Protothreads - lightweight stackless threads in c, 2005. 
$\left[\mathrm{DZK}^{+} 02\right]$ Frank Dabek, Nickolai Zeldovich, Frans Kaashoek, David Mazieres, and Robert Morris. Event-driven programming for robust software. In Proceedings of the 10th ACM SIGOPS European Workshop, September 2002.

[Fee00] M. Feeley. A portable implementation of first-class continuations for unrestricted interoperability with $\mathrm{c}$ in a multithreaded scheme, 2000.

[Fer06] Stephen Ferg. Event-Driven Programming: Introduction, Tutorial, History. 2006.

[Gra04] Paul Graham. onlisp. 2004.

[Knu73] Donald E. Knuth. Fundamental Algorithms, volume 1 of The Art of Computer Programming, section 1.2, pages 10119. Addison-Wesley, Reading, Massachusetts, second edition, 10 January 1973. This is a full INBOOK entry.

[Kor01] Leif Kornstaedt. Alice in the land of $\mathrm{Oz}-$ an interoperabilitybased implementation of a functional language on top of a relational language. In Proceedings of the First Workshop on Multilanguage Infrastructure and Interoperability (BABEL'01), Electronic Notes in Computer Science, volume 59, Firenze, Italy, September 2001. Elsevier Science Publishers.

[MEG94] Bodhisattwa Mukherjee, Greg Eisenhauer, and Kaushik Ghosh. A machine independent interface for lightweight threads. $A C M$ Operating Systems Review, 28(1):33-47, 1994.

[MPT17] Juan David Arcila Moreno, Santiago Passos, and Mauricio Toro. On-line assembling mitochondrial DNA from de novo transcriptome. CoRR, abs/1706.02828, 2017.

[MTC18] Raul Mazo, Mauricio Toro, and Luz Cobaleda. Definicion de la arquitectura de referencia de un dominio: de la elucidacion al modelado. In Raul Mazo, editor, Guia para la adopcion industrial de lineas de productos de software, pages 193-210. Editorial Eafit, 2018.

[ORS $\left.{ }^{+} 11\right]$ Carlos Olarte, Camilo Rueda, Gerardo Sarria, Mauricio Toro, and Frank Valencia. Concurrent Constraints Models of Music Interaction. In Gérard Assayag and Charlotte Truchet, editors, 
Constraint Programming in Music, chapter 6, pages 133-153. Wiley, Hoboken, NJ, USA., 2011.

[PFATT16] C. Patiño-Forero, M. Agudelo-Toro, and M. Toro. Planning system for deliveries in Medellín. ArXiv e-prints, November 2016.

[PS06] Peterson and Silverchatz. Operating Systems Concepts. 2006.

[PT13] Anna Philippou and Mauricio Toro. Process Ordering in a Process Calculus for Spatially-Explicit Ecological Models. In Proceedings of MOKMASD'13, LNCS 8368, pages 345-361. Springer, 2013.

[PTA13] Anna Philippou, Mauricio Toro, and Margarita Antonaki. Simulation and Verification for a Process Calculus for SpatiallyExplicit Ecological Models. Scientific Annals of Computer Science, 23(1):119-167, 2013.

[QSRT18] E. Quintero, M. Sanchez, N. Roldan, and M. Toro. Genetic algorithm for optimal distribution in cities. ArXiv e-prints, November 2018.

[Ree05] Glenn Reeves. What really happend on mars, 2005.

[RHRS ${ }^{+}$18] Cristian Rendon, José L Hernández, Oscar Ruiz-Salguedo, Carlos A Álvarez, and Mauricio Toro. Wing Profile Evolution driven by Computational Fluid Dynamics. In Tecnologías Avanzadas de Mecatrónica, Diseño y Manufactura: Memorias Cuarto Congreso AMDM, pages 345-347. Editorial Universidad Autónoma de Manizales, 2018.

[Roy08] Peter Van Roy. Reply to email about lightweight threads, 2008.

[RPT17] Juan Manuel Ciro Restrepo, Andrés Felipe Zapata Palacio, and Mauricio Toro. Assembling sequences of DNA using an on-line algorithm based on debruijn graphs. CoRR, abs/1705.05105, 2017.

[Sch02] Christian Schulte. Programming Constraint Services: HighLevel Programming of Standard and New Constraint Services, volume 2302. Springer, 2002. 
[Shi97] O. Shivers. Continuations and threads: Expressing machine concurrency directly in advanced languages, 1997.

[SMP91] V. Saraswat, M.Rinard, and P.Panangaden. The semantic foundations of concurrent constraint programming, 1991.

[SS92] Dan Stein and Devang Shah. Implementing lightweight threads. In Proceedings of theSummer 1992 USENIX Technical Conference and Exhibition, pages 1-10, San Antonio, TX, 1992. USENIX.

$\left[\mathrm{STM}^{+} 18\right]$ Elizabeth Suescún, Mauricio Toro, Raul Mazo, David Velasquez, Paola Vallejo, Juan F. Cardona, Rafael Rincón, Vera Maria Werneck, and Julio Cesar Camapio do Prado Leite. SimulES-W: A Collaborative Game to Improve Software Engineering Teaching. Computación y Sistemas, 22(3):953-983, 2018.

[TAAR09] Mauricio Toro, Carlos Agón, Gérard Assayag, and Camilo Rueda. Ntccrt: A concurrent constraint framework for realtime interaction. In Proc. of ICMC '09, Montreal, Canada, 2009.

[Tac08] Guido Tack. Reply to email about lightweight threads in seam, 2008.

[TDC10] Mauricio Toro and Myriam Desainte-Catherine. Concurrent constraint conditional branching interactive scores. In Proc. of $S M C$ '10, Barcelona, Spain, 2010.

[TDCB10] Mauricio Toro, Myriam Desainte-Catherine, and P. Baltazar. A model for interactive scores with temporal constraints and conditional branching. In Proc. of Journées d'Informatique Musical (JIM) '10, May 2010.

[TDCC12] Mauricio Toro, Myriam Desainte-Catherine, and Julien Castet. An extension of interactive scores for multimedia scenarios with temporal relations for micro and macro controls. In Proc. of Sound and Music Computing (SMC) '12, Copenhagen, Denmark, July 2012.

[TDCC16] MAURICIO TORO, MYRIAM DESAINTE-CATHERINE, and JULIEN CASTET. An extension of interactive scores 
for multimedia scenarios with temporal relations for micro and macro controls. European Journal of Scientific Research, 137(4):396-409, 2016.

[TDCJO18] Mauricio Toro, Myriam Desainte-Catherine, David Janin, and Yann Orlarey. Real-time interactive streams and temporal objects language. OSF Preprints, 10.31219/osf.io/a6stk, Oct 2018.

[TDCR14] Mauricio Toro, Myriam Desainte-Catherine, and Camilo Rueda. Formal semantics for interactive music scores: a framework to design, specify properties and execute interactive scenarios. Journal of Mathematics and Music, 8(1):93-112, 2014.

[Tor08] Mauricio Toro. Exploring the possibilities and limitations of concurrent programming for multimedia interaction and graphical representations to solve musical csp's. Technical Report 2008-3, Ircam, Paris.(FRANCE), 2008.

[Tor09a] Mauricio Toro. Probabilistic Extension to the Factor Oracle Model for Music Improvisation. Master's thesis, Pontificia Universidad Javeriana Cali, Colombia, 2009.

[Tor09b] Mauricio Toro. Towards a correct and efficient implementation of simulation and verification tools for probabilistic ntcc. Technical report, Pontificia Universidad Javeriana, May 2009.

[Tor10] Mauricio Toro. Structured interactive musical scores. In Manuel V. Hermenegildo and Torsten Schaub, editors, Technical Communications of the 26th International Conference on Logic Programming, ICLP 2010, July 16-19, 2010, Edinburgh, Scotland, UK, volume 7 of LIPIcs, pages 300-302. Schloss Dagstuhl - Leibniz-Zentrum fuer Informatik, 2010.

[Tor12] Mauricio Toro. Structured Interactive Scores: From a simple structural description of a multimedia scenario to a real-time capable implementation with formal semantics. PhD thesis, Univeristé de Bordeaux 1, France, 2012.

[Tor15] Mauricio Toro. Structured interactive music scores. CoRR, abs/1508.05559, 2015.

[Tor16a] M. Toro. Probabilistic Extension to the Concurrent Constraint Factor Oracle Model for Music Improvisation. ArXiv e-prints, February 2016. 
[Tor16b] Mauricio Toro. Probabilistic Extension to the Concurrent Constraint Factor Oracle Model for Music Improvisation . Inteligencia Artificial, 57(19):37-73, 2016.

[Tor18a] M. Toro. Current Trends and Future Research Directions for Interactive Music. ArXiv e-prints, October 2018.

[Tor18b] M. Toro. Towards a correct and efficient implementation of simulation and verification tools for probabilistic ntcc. ArXiv e-prints, October 2018.

[Tor18c] Mauricio Toro. Backtracking-free interactive music scores with temporal relations over rythms. OSF Preprints, 10.31219/osf.io/bwzsk, Oct 2018.

[Tor18d] Mauricio Toro. Combining temporal relations and signal processing into interactive scores. OSF Preprints, osf.io/sqmer, Oct 2018 .

[Tor18e] Mauricio Toro. CURRENT TRENDS AND FUTURE RESEARCH DIRECTIONS FOR INTERACTIVE MUSIC. Journal of Theoretical and Applied Information Technology, 69(16):5569-5606, 2018.

[Tor18f] Mauricio Toro. Modeling michael jarrell's csp in gelisp. OSF Preprints, osf.io/2ebzj, Oct 2018.

[Tor18g] Mauricio Toro. ntccmc: A bounded-time model checker for ntcc. OSF Preprints, osf.io/c8kah, Oct 2018.

[Tor18h] Mauricio Toro. Towards a correct and efficient implementation of simulation and verification tools for probabilistic ntcc. $O S F$ Preprints, 10.31219/osf.io/3wp5z, Oct 2018.

[TPA ${ }^{+}$16] Mauricio Toro, Anna Philippou, Sair Arboleda, María Puerta, and Carlos M. Vélez S. Mean-field semantics for a process calculus for spatially-explicit ecological models. In César A. Muñoz and Jorge A. Pérez, editors, Proceedings of the Eleventh International Workshop on Developments in Computational Models, Cali, Colombia, October 28, 2015, volume 204 of Electronic Proceedings in Theoretical Computer Science, pages 79-94. Open Publishing Association, 2016. 
[TPKS14] Mauricio Toro, Anna Philippou, Christina Kassara, and Spyros Sfenthourakis. Synchronous parallel composition in a process calculus for ecological models. In Gabriel Ciobanu and Dominique Méry, editors, Proceedings of the 11th International Colloquium on Theoretical Aspects of Computing - ICTAC 2014, Bucharest, Romania, September 17-19, volume 8687 of Lecture Notes in Computer Science, pages 424-441. Springer, 2014.

[TRAA15] MAURICIO TORO, CAMILO RUEDA, CARLOS AGÓN, and GÉRARD ASSAYAG. Ntccrt: A concurrent constraint framework for soft real-time music interaction. Journal of Theoretical G Applied Information Technology, 82(1), 2015.

[TRAA16] MAURICIO TORO, CAMILO RUEDA, CARLOS AGÓN, and GÉRARD ASSAYAG. Gelisp: A framework to represent musical constraint satisfaction problems and search strategies. Journal of Theoretical \&6 Applied Information Technology, 86(2), 2016.

[TRG $\left.{ }^{+} 87\right]$ Avadis Tevanian, Jr., Richard F. Rashid, David B. Golub, David L. Black, Eric Cooper, and Michael W. Young. Mach threads and the unix kernel: The battle for control. Technical Report CMU-CS-87-149, 1987.

[Uni03] Raphael Collet Universit'e. Laziness and declarative concurrency, 2003.

[Vah96] Uresh Vahalia. UNIX Internals. 1996.

[vBCB03] R. von Behren, J. Condit, and E. Brewer. Why events are a bad idea for high-concurrency servers, 2003.

[VH04] Peter Van Roy and Seif Haridi. Concepts, Techniques, and Models of Computer Programming. MIT Press, March 2004.

[WGQH] B. Weissman, B. Gomes, J. Quittek, and M. Holtkamp. Efficient fine-grain thread migration with active threads. pages 410-414. 
Figure 3: CCP interpreter using Event Driven Programming

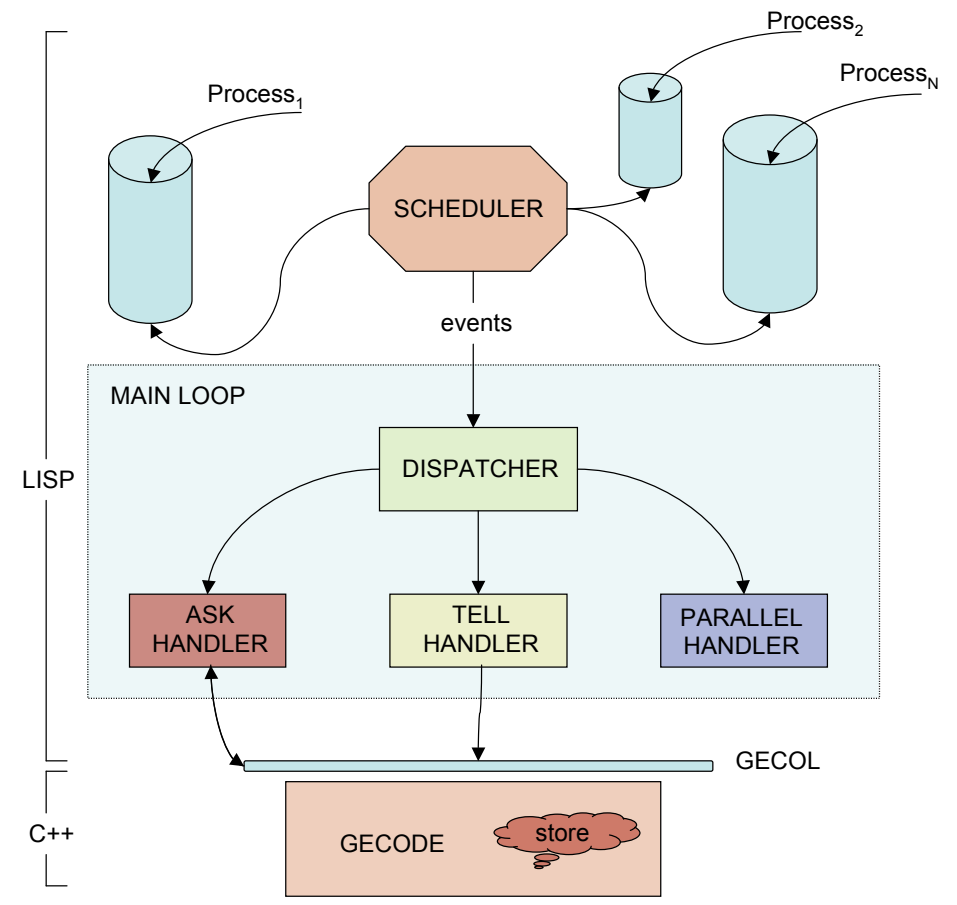

